\title{
Dedicated Magnetic Resonance Imaging Sequences: Contribution in the Diagnosis of Focal Epilepsy in the Lebanese Population
}

\author{
Najo A. Jomaa ${ }^{1,3}$, Marwan Haddad ${ }^{1,2}$, Grace Y. Adwane ${ }^{1,3}$, Amira J. Zaylaa ${ }^{1,4}$ and Abdallah Rahbani ${ }^{1,3}$ \\ 1. Faculty of Medical Sciences, Lebanese University, Hadath Baabda 1003, Lebanon \\ 2. Imaging Department, Mount Lebanon Hospital, Hazmieh 1107, Lebanon \\ 3. Epilepsy monitoring unit, Department of Neurology, Lebanese Geitaoui University Hospital, Achrafieh Beirut 1100, Lebanon \\ 4. Doctoral School of Science and Technology, Lebanese University, Hadath Baabda 1003, Lebanon
}

\begin{abstract}
There have been many advances in the diagnosis and management of focal epilepsies particularly with neuroimaging techniques and magnetic resonance imaging (MRI). Special MRI sequences can be employed to localize and resect the epileptogenic lesions responsible for focal epilepsy. This study aims to show the benefit of dedicated epilepsy MRI sequences in the diagnosis of focal epilepsies. A general electric 1.5 Tesla MRI machine was used with standard and special sequences. Also, a Nihon Kohden electroencephalography (EEG) machine was used. This is a prospective observational study that included 51 patients with focal epilepsies who had an initial negative brain imaging. They underwent epilepsy MRI sequences along with a prolonged video EEG monitoring to localize the lesion, and then results were analyzed statistically using SPSS 22 program. The majority of patients were males (62.75\%) with a mean age of 30 years. The grand majority of patients (74.5\%, $p$ value of 0.001$)$ had their lesion localized by the epilepsy MRI. The most commonly found pathology was mesial temporal sclerosis. A significant number of patients (23.5\%) were sent for an epilepsy surgery ( $p$ value 0.002 ). This study shows the significant impact of dedicated epilepsy MRI sequences on the diagnosis and management of focal epilepsy in the Lebanese population.
\end{abstract}

Key words: Epilepsy, magnetic resonance imaging, dedicated sequences, focal, epileptogenic lesions, hippocampal sclerosis, cortical dysplasia.

\section{Introduction}

During the last decade, there have been many advances in our understanding and management of epilepsies. With the emerging neuroimaging modalities including specialized magnetic resonance imaging (MRI) sequences, the total number of presumed non-idiopathic cryptogenic partial epilepsies is drastically decreasing [1, 2].

An epileptic seizure is defined as a transient alteration in mentation associated with abnormal movements as a result of excessive bursts of brain electrical activity [3]. However, epilepsy itself is a chronic condition in which seizures recur, and this can

Corresponding author: Abdallah Rahbani, M.D., associate professor, research fields: neurology and neurophysiology. be complicated by neurobiological, cognitive, psychosocial, and occupational consequences [4, 5].

The 2017 International League Against Epilepsy (ILAE) classification divides epilepsy into 3 types: those with focal onset, generalized onset, and unknown onset. Focal seizures were further divided into those with and without an impaired awareness [3]. Focal epilepsy is caused by an abnormal electrical discharge from an epileptogenic zone, which is the cortical area indispensable for the seizure generation [6].

The majority of epileptic patients are satisfactorily treated medically with antiepileptic drugs, but around $15-30 \%$ continue to have seizures despite therapy $[2,4,5]$.

Epilepsy is usually considered to be refractory if it cannot be controlled after the use of multiple drugs for 
more than 2 years [7, 8]. In this case, surgery is a major therapeutic alternative that can render $70 \%$ of patients seizure free $[4,7,9]$. This is much easier to obtain when the lesion is well identified on brain imaging prior to the surgery [6].

Thus, neuroimaging is an essential step in the presurgical workup of localization-related epilepsies in order to well delineate the epileptogenic zone $[2,7]$.

MRI is considered to be the imaging modality of choice in defining the structural lesions that are responsible for seizures [5]. This is due to its better soft tissue contrast, multiplanar imaging and safety profile $[2,10]$.

Earlier studies have shown that around $20 \%$ of epilepsies have no lesion that is identified on MRI [1, 11]. However, a study done on children having refractory epilepsy found that a high-resolution MRI was able to detect lesions not previously detected on a standard MRI in more than half of the cases [2].

Important pathologies that must be addressed in focal epilepsy include Mesial Temporal Sclerosis (MTS), Focal Cortical Dysplasias (FCD), developmental cortical malformations, arteriovenous malformation, tumors and infections [5, 6, 12-14]. Of these underlying pathologies, MTS is the most common entity in the drug-resistant epilepsy [15].

In this regard, dedicated MRI sequences have been developed. These include high-resolution thin cuts using coronal FLAIR, T2, T2* Gradient Echo and 3D sequences [4, 16-18].

The role of Electroencephalography (EEG) is also a major one as the presence of a lesion on MRI does not necessarily indicate its epileptogenicity, unless the result is congruent with the EEG findings [2].

It is important to note that many patients with epilepsy frequently undergo standard MRI or CT in their initial workup in non-specialized centers and many of them have a missed diagnosis [9]. In Lebanon and other developing countries, this scenario is of a higher occurrence because of the limited resources in the management of epilepsy [10].
The aim of our study is to show the additional value of dedicated epilepsy MRI sequences in the detection of brain lesions in patients having cryptogenic focal epilepsy in the Lebanese population. Our study will hopefully guide the medical professionals to do a more extended work-up of the patients presenting with focal seizures.

\section{Materials}

The materials we used in this study are mentioned below. These included also the different types of MRI sequences we used for imaging.

(1) A 1.5 Tesla MRI machine (General Electric (GE) optima) with the following sequences:

A. Standard MRI Sequences

- Axial T2 Fluid attenuation recovery (FLAIR) sequence (4 $\mathrm{mm}$ slice, $1 \mathrm{~mm}$ interspace gap);

- Axial T2 sequence (4 mm slice, $1 \mathrm{~mm}$ interspace gap);

- Axial diffusion weighted image (DWI) 1000b

B. Specific Additional Epilepsy Sequences

- Coronal T1 inversion recovery;

- Coronal T2 FLAIR (thin 3 mm slice, no interspace gap);

- SWI sequence (called SWAN in GE MRI machine) (thin $3 \mathrm{~mm}$ slice);

- Sagittal Fast Spoiled Gradient Recall 3D (FSPGR) sequence (thin $1.2 \mathrm{~mm}$ slice thickness with no intervening gap).

In case a neoplasm or neurocutaneous syndrome was suspected a contrast agent was injected intravenously: Gadodiamide (Omniscan) at a dose of $0.1 \mathrm{mmol} / \mathrm{kg}$ of body weight.

(2) A 128-channel video-EEG monitoring machine in an epilepsy monitoring unit. It is used as a part of a comprehensive epilepsy program at the Lebanese Geitaoui University Hospital.

\section{Methods}

According to our exploratory study, we took into consideration the following patients demographics, 
ethical considerations and data collection methods.

\subsection{Patients Demographics}

This is a prospective study that included patients of all ages whether males or females, having focal epilepsy with a negative initial brain imaging.

Patients were enrolled in the study according to the following inclusion and exclusion criteria.

Inclusion Criteria:

- Partial epilepsy;

- Non-idiopathic;

- Negative baseline neuroimaging (CT scan or routine MRI).

Exclusion Criteria:

- Generalized epilepsy;

- Idiopathic;

- Positive baseline imaging for a pathologic lesion.

\subsection{Ethical Consideration}

Patients included in this study were not subjected to any new procedure or medication. They underwent the basic exams that comprehensive epilepsy programs would include in order to diagnose and treat epilepsy. Thus subjects were not at risk of harm by any step of the study. Besides, confidential and private information concerning the patients was not exposed to anyone else but the treating physician.

\subsection{Collection of Data}

This study is a prospective observational study in which we included 51 patients presenting with focal epilepsy and a negative baseline brain imaging (CT scan or MRI). These patients were enrolled over a period of around 5 months extending from April to August 2017.

All the patients underwent an initial prolonged video-EEG monitoring in the setting of a university hospital and were included in a comprehensive epilepsy program. This was done with the aim to detect a clinical seizure or electrical epileptiform discharges. Then, patients were referred to a specialized radiology department to do dedicated MRI epilepsy sequences. MRI sequences were analyzed by the radiologists to detect a focal pathologic lesion. These results were then compared to the initial imaging results in order to analyze statistically the significance of these specialized sequences in focal epilepsy. Finally a PET scan was ordered for those patients who had a negative dedicated MRI with a high suspicion for a focal lesion (See Fig. 1).

\subsubsection{Statistical Analysis Method}

In our study, all the statistical analysis was carried out with SPSS Statistics version 22 (IBM, Inc, Armonk, NY). We calculated the mean only for the age. For the other categorical data, we calculated frequencies and

1- Patients undergo prolonged video-EEG monitoring.

2- Patients are referred to do dedicated epilepsy MRI sequences.

3- Dedicated MRI is analyzed by specialized radiologists.

4- Results of dedicated MRI are compared to results of initial brain imaging (MRI or CT scan) and evaluated statistically.

5- Patients with negative dedicated MRI are referred for PET scan.

Fig. 1 Steps followed in the methodology of this study. 
percentages. For the $p$-value, we used a one sample t-test where a p-value of less than 0.05 was considered to be significant.

\section{Results}

In this section below, the different results regarding the distribution of the types of seizures, pathologies, and their correlation with MRI results in addition to the different patients' characteristics will be presented.

\subsection{Patients' Characteristics}

A total of 51 patients with focal epilepsy were included in this study. Our patients were divided into 32 males (62.7\%) and 19 females (37.2\%). Ages ranged between 2 years and 85 years with a mean age of 30 years.

\subsection{Types of Seizures}

Upon presentation, the majority of patients had temporal electrical discharges on the EEG (37.25\%), followed in frequency by frontal discharges (21.56\%). Moreover, 7 patients out of 51 had multifocal discharges (13.73\%) and 3 patients had epileptic encephalopathy on the EEG (5.88\%) (Table 1).

\subsection{Epilepsy MRI Results}

\subsubsection{Positive MRI Results}

Out of the 51 patients included in the study, 38 patients $(74.5 \%)$ had a confirmed diagnosis by an epilepsy MRI which is statistically significant ( $p$-value $=0.001$ ). In these patients, specialized MRI sequences were able to detect the underlying epileptogenic lesion responsible for their epilepsy.

\subsubsection{Types of Pathologies}

The majority of lesions that were detected on the specialized MRI consisted mainly of hippocampal sclerosis and cortical dysplasias. A wide variety of other lesions were also detected ranging from congenital abnormalities such as heterotopias and polymicrogyrias to low grade tumors such as DNET and gliomas (Table 2).
One of the patients included in the study was found to have a dual pathology on imaging. These consisted of hippocampal sclerosis and focal cortical dysplasia (Figs. 2 and 3).

The majority of the detected pathologies, as shown in Table 3, are non-neoplastic.

Table 3 demonstrates the difference in number between congenital and acquired causes. As shown below, the majority of pathologies were consisting of acquired causes.

\subsection{Age Distribution versus Pathology}

The great majority of patients who were found to have a focal cortical dysplasia in our study were in the

Table 1 Distribution of the types of seizures or discharges present on EEG.

\begin{tabular}{lll}
\hline Type of seizure & $\begin{array}{l}\text { Number of studied } \\
\text { EEGs }(n=51)\end{array}$ & Percentage \% \\
\hline Temporal & 19 & 37.25 \\
Frontal & 11 & 21.56 \\
Multifocal & 7 & 13.73 \\
Parietal & 6 & 11.76 \\
Epileptic encephalopathy & 3 & 5.88 \\
Occipital & 2 & 3.92 \\
Gelastic & 1 & 1.96 \\
Psychomotor & 1 & 1.96 \\
Insular & 1 & 1.96 \\
\hline
\end{tabular}

Table 2 Different types of epileptogenic lesions detected on specialized MRI.

\begin{tabular}{lll}
\hline Type of lesion & $\begin{array}{l}\text { Number of studied } \\
\text { specialized MRIs } \\
(n=38)\end{array}$ & Percentage \% \\
\hline Hippocampal sclerosis & 10 & 26.3 \\
Cortical dysplasias & 9 & 23.7 \\
Infarct with gliosis & 4 & 10.5 \\
DNET & 3 & 7.9 \\
Polymicrogyria & 2 & 5.3 \\
Tuberous sclerosis & 2 & 5.3 \\
Low grade glioma & 2 & 5.3 \\
Meningioma & 1 & 2.6 \\
Hypothalamic hamartoma & 1 & 2.6 \\
Sturge weber & 1 & 2.6 \\
Posterior reversible & & \\
encephalopathy syndrome & 1 & 2.6 \\
(PRES) & & \\
Heterotopia & 1 & 2.6 \\
Porencephalic cavity & 1 & 2.6 \\
\hline
\end{tabular}




\section{Focal Epilepsy in the Lebanese Population}

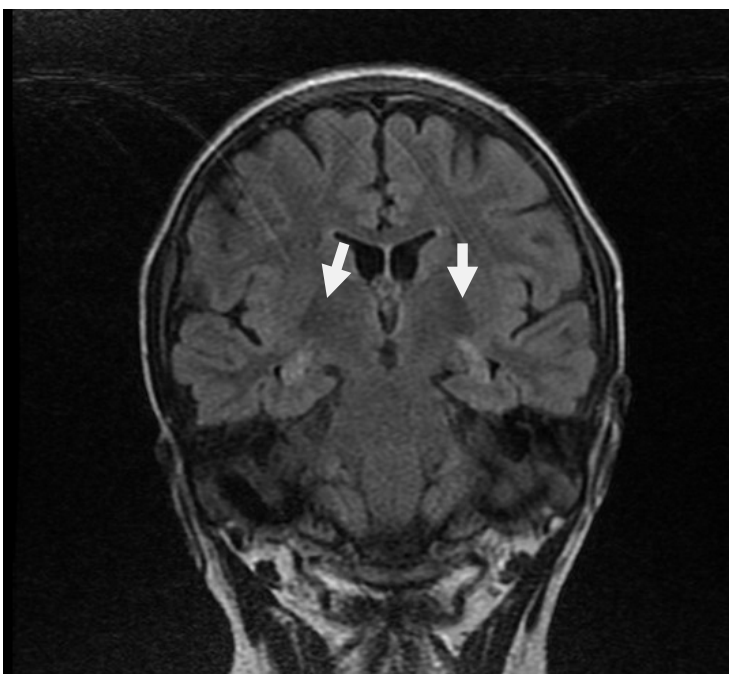

Fig. 2 A coronal FLAIR sequence with thin cuts showing bilateral hippocampal sclerosis (white arrows).

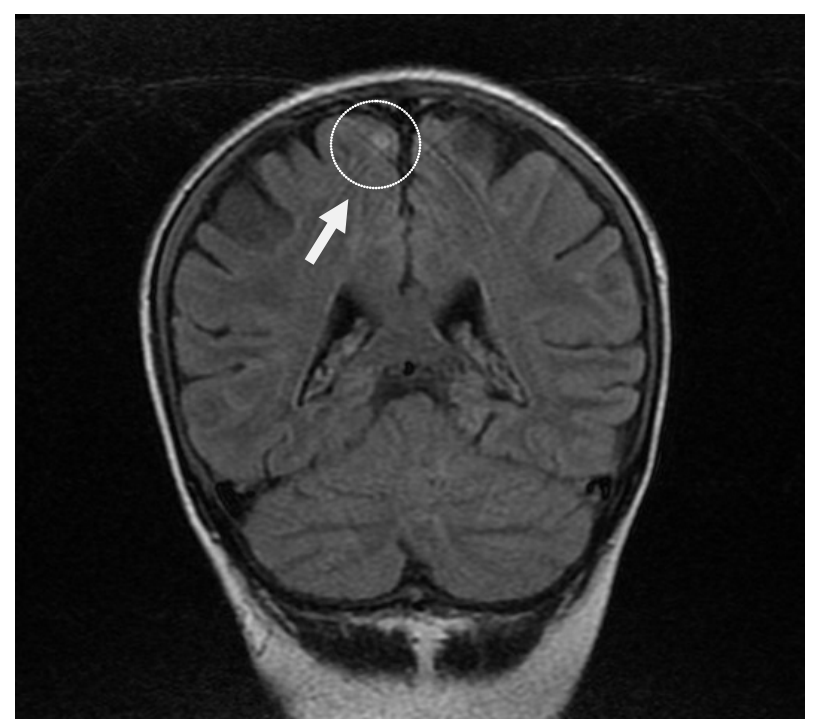

Fig. 3 A coronal FLAIR sequence with thin slices in the same patient showing a hyperintense cortical lesion most probably a focal cortical dysplasia (white arrow and white outline).

Table 3 The distribution of the detected lesions between neoplastic, non-neoplastic etiologies, congenital and acquired causes.

\begin{tabular}{lll}
\hline Type of etiology & $\begin{array}{l}\text { Number of studied } \\
\text { etiologies }(n=38)\end{array}$ & Percentage \% \\
\hline Neoplastic & 7 & 18.42 \\
Non-neoplastic & 31 & 81.58 \\
Congenital & 15 & 39.47 \\
Acquired & 23 & 60.53 \\
\hline
\end{tabular}

first or second decade, while almost all of the patients with mesial temporal sclerosis were above 20 years of age.

\subsection{Positron Emission Tomography (PET) Scan}

Four patients out of the 12 cases with a negative MRI underwent PET scan and were shown to have an epileptogenic lesion. One of these underwent surgical resection of the lesion and another patient underwent a VNS (Vagal Nerve Stimulation) surgery.

\subsection{Disease Management}

Thirteen patients were sent for an epileptic surgery based on their dedicated MRI results (12 patients, 23.5\%), and 3 patients were sent for VNS (5.8\%). Almost all of these patients are still seizure free postoperatively. Another larger group of patients (22 patients, 43.1\%) with positive MRI were managed with an appropriate medical treatment that was suitable for their underlying lesion.

\section{Discussion}

Epilepsy is still one of the major challenging Central Nervous System (CNS) disorders. Reaching a definite diagnosis has a key value for the prognosis and therapeutic decisions.

This is largely dependent on the clinical history and findings, along with a dedicated and specialized neuroimaging and electrophysiologic procedures.

Our results showed that when the clinical picture was highly in favor of a non-idiopathic focal epilepsy, dedicated MRI sequences for epilepsy were able to well localize epileptogenic lesions in $74.51 \%$ of the studied patient population in whom the basic neuroimaging studies were not conclusive. This number is considered significant (with a $p$-value of 0.001 ) and it is associated with a large impact on the diagnostic and therapeutic approach of epilepsy patients, especially in the Lebanese population.

This fact was highlighted by the significant number of patients who underwent a major change in their therapeutic strategy. This mainly consisted of epilepsy surgery and VNS.

It is important to note that patients were selected for surgery based on many factors including the 
operability of the lesion (whether it is present in an eloquent cortex or not) in addition to the validity and correlation of the clinical, radiological, and electrophysiological findings, i.e. patients lacking this correlation were considered for much further work up (for example PET scan) and were treated medically.

Our results were congruent with the previous studies showing that mesial temporal sclerosis is still the most frequent pathology detected on brain MRI in focal epilepsy patients. Also similar to previous studies, the majority of the detected lesions were acquired (60.53\%) and the majority of these too were non-neoplastic (81.55\%).

Our results showed the interest of asking for an epilepsy protocol MRI in patients presenting with seizures (especially in those presenting with focal seizures) by their primary care physicians, neurologists and epileptologists.

This is important considering the fact that many patients in our country Lebanon and other developing countries are not undergoing this basic step in managing their epilepsy. This is mainly due to lack of insight on the importance of these very specific techniques, or due to the lack of financial medical coverage of patients. Many patients for example seek medical care and imaging in nonspecialized centers for financial reasons.

On the other hand, many centers in Lebanon are already equipped with high resolution MRI machines that are able to perform all the previously mentioned specialized epilepsy sequences. This is in addition to the fact that also a large number of radiologists are very well trained in this regard.

\section{Conclusion}

Refractory seizures are a major cause of morbidity in epileptic patients causing devastating consequences on several aspects of their lives. Knowing that a significant number of epileptic patients have focal epilepsy makes it very important to well localize the causative epileptogenic lesion. This would help in referring patients for a more effective surgical therapy.

This study showed the large influence a dedicated epilepsy MRI can have in the diagnosis and management of epileptic patients with focal epilepsy.

This study showed that a large number of patients in the Lebanese population can be falsely diagnosed as having cryptogenic epilepsy with an unknown causative lesion.

In this regard, it is important to send these patients to do a specific epilepsy protocol MRI instead of nonspecific brain imaging in their initial work up.

\section{References}

[1] Degnan, A. J., et al. 2014. "Neuroimaging of Epilepsy: A Review of MRI Findings in Uncommon Etiologies and Atypical Presentations of Seizures.” Future Neurology 9: 431-48.

[2] Ramli, N., 2015. et al. "Neuroimaging in Refractory Epilepsy: Current Practice and Evolving Trends." European Journal of Radiology, March 2015.

[3] Fisher, R. S., et al. 2017. "The New Classification of Seizures by the International League Against Epilepsy 2017." Current Neurology and Neuroscience Reports 17: 48.

[4] Athira, D., et al. 2015. "Role of Magnetic Resonance Imaging Brain in Evaluation of Seizures." Journal of Evolution of Medical and Dental Science 78: 13654-70.

[5] Hartman, L. A., et al. 2015. "Epilepsy Imaging: Approaches and Protocols." Applied Radiology. www.appliedradiology.com: 8-20.

[6] Friedman, E. 2014. "Epilepsy Imaging in Adults: Getting It Right.” AJR 203:1093-103.

[7] Saini, J., et al. 2009. "Susceptibility Weighted Imaging in the Diagnostic Evaluation of Patients with Intractable Epilepsy.” Epilepsia 50: 1462-73.

[8] French, J. A. 2007. "Refractory Epilepsy: Clinical Overview.” Epilepsia 48: 3-7.

[9] VonOertzen, J., et al. 2002. "Standard Magnetic Resonance Imaging Is Inadequate for Patients with Refractory Focal Epilepsy.” J NeuoNeurosurg Psychiatry 73: 643-47.

[10] Chang, R. S., et al. 2016. “Call for Quality Epilepsy Neuroimaging-An Illustrative Case of Missed Epileptic Substrate.” Journal of the Formoscan Medical Association 115: 814-5.

[11] Tellez-Zenteno, J. F., et al. 2010. "Surgical Outcomes in Lesions and Nonlesional Epilepsy: A Systemic Review and Meta-Analysis.” Epilepsy Res 89: 310-8. 


\section{Focal Epilepsy in the Lebanese Population}

[12] Colombo, N., et al. 2003. "Focal Cortical Dysplasias: MR Imaging, Histopathologic, and Clinical Correlation in Surgically Treated Patients with Epilepsy.” AJNR 24: 724-33.

[13] Colombo, N., et al. 2012. "Focal Cortical Dysplasia Type IIa and IIb: MRI Aspects in 118 Cases Proven by Histopathology.” Neuroradiology 54: 1065-77.

[14] Turjman, F., et al. 1995. "Epilepsy Associated with Cerebral Arteriovenous Malformations: A Multivariate Analysis of Angioarchitectural Characteristics.” AJNR 16: 345-50.
[15] Zentner, J., et al. 1999. "Clinical Relevance of Amygdala Sclerosis in Temporal Lobe Epilepsy.” $J$ Neurosurg 91: 59-67.

[16] Hasboun, D., et al. 1996. "MR Determination of Hippocampal Volume: Comparison of Three Methods.” AJNR 17: 1091-8.

[17] Urbach, H., et al. 2004. "MR Imaging in the Presurgical Workup of Patients with Drug-Resistant Epilepsy.” AJNR 25: 919-26.

[18] Urbach, H., et al. 2005. "Imaging of the Epilepsies." EurRadiol 15: 494-500. 